\title{
DESEMPENHO AGRONÔMICO DO RABANETE EM FUNÇÃO DE LÂMINAS DE IRRIGAÇÃO E NÍVEIS DE ADUBAÇÃO NITROGENADA
}

\author{
Julianna Catonio da Silva ${ }^{1 *}$, Felipe dos Santos Barbosa², Franklin Correia Lima², Kaio Cesar Santos², Wirlan \\ Farias dos Santos ${ }^{2}$, Márcio Aurélio Lins dos Santos ${ }^{3}$
}

\begin{abstract}
'Engenheira Agrônoma, Doutoranda em Produção Vegetal, Universidade Federal de Alagoas (UFAL), BR 104 Norte, Km 85, Rio Largo/ AL- CEP: $57.100-000$

${ }^{2}$ Graduandos em Agronomia, Universidade Federal de Alagoas (UFAL), Campus Arapiraca, Av. Manoel Severino Barbosa, s/n - Bom Sucesso, Arapiraca/AL - CEP: 57.309-005

${ }^{3}$ Professor Doutor, Universidade Federal de Alagoas (UFAL), Campus Arapiraca, Av. Manoel Severino Barbosa, s/n - Bom Sucesso, Arapiraca/AL - CEP: 57.309-005
\end{abstract}

*Autor para correspondência: Julianna Catonio da Silva, julianna_cds@hotmail.com

RESUMO: Dos fatores de produção, a água e os nutrientes são aqueles que limitam a produtividade da cultura do rabanete com maior intensidade. Desse modo, o controle da irrigação e da fertilidade do solo são critérios preponderantes para o êxito da agricultura. Objetivou-se determinar o desempenho agronômico do rabanete em função de lâminas de irrigação e níveis de adubação nitrogenada. 0 experimento foi conduzido em ambiente protegido na Universidade Federal de Alagoas, Campus de Arapiraca, no período de abril a maio de 2017. Foi utilizado o delineamento experimental em blocos ao acaso, num esquema fatorial $5 \times 5$, com 4 blocos, totalizando 100 parcelas experimentais. A unidade experimental foi representada por um recipiente plástico com área superficial de $0,0314 \mathrm{~m}^{2}$, com capacidade máxima para $5 \mathrm{~L}$. As variáveis analisadas foram: número de folhas (NF), diâmetro do bulbo (DB), massa fresca da parte aérea (MFPA), massa seca da parte aérea (MSPA) e produtividade (P). A lâmina de $150 \%$ da ETc proporcionou os melhores resultados para as variáveis analisadas e apenas a MFPA apresentou efeito positivo ao aumento das doses de nitrogênio.

PALAVRAS-CHAVE: Raphanus sativus L.; água; nutrientes.

\section{AGRONOMIC PERFORMANCE OF THE RADIANT AS A FUNCTION OF IRRIGATION BLADES AND NITROGENATED FERTILIZATION LEVELS}

\begin{abstract}
Of the factors of production, water and nutrients are those that limit the productivity of the radish crop with greater intensity. Thus, the control of irrigation and soil fertility are preponderant criteria for the success of agriculture. The objective was to determine the agronomic performance of the radish as a function of irrigation depths and nitrogen fertilization levels. The experiment was conducted in a protected environment at the Federal University of Alagoas, Campus de Arapiraca, from April to May 2017. A randomized block design was used, in a $5 \times 5$ factorial scheme, with 4 blocks, totaling 100 experimental plots. The experimental unit was represented by a plastic container with a surface area of $0.0314 \mathrm{~m} 2$, with a maximum capacity of $5 \mathrm{~L}$. The variables analyzed were: number of leaves (NF), bulb diameter (DB), fresh shoot weight (MFPA), dry shoot weight (MSPA) and productivity (P). The $150 \%$ ETc blade provided the best results for the variables analyzed and only the MFPA had a positive effect on the increase in nitrogen doses.
\end{abstract}

KEYWORDS: Raphanus sativus L.; Water; nutrients.

\section{INTRODUÇÃO}

O rabanete (Raphanus sativus $L$.) é uma brássica que possui vitaminas $A, B 1, B 2, B 6, C$, ácido fólico, sais minerais (potássio, cálcio, fósforo e enxofre), além de baixa quantidade de calorias (Matos et al., 2019).
Pertence a mesma família da Brassica oleracea (couve, brócolis, repolho e couve-flor), só que diferente destas a parte consumida é uma raiz tuberosa (Lima et al., 2017). Apresenta pequena importância em termos de área cultivada, entretanto, possui boa viabilidade econômica. 
A irrigação do rabanete, além de constituir um importante fator de produção, favorece 0 aumento da produtividade, bem como o aprimoramento da qualidade do produto (Pereira et al., 2018). Para tanto, é necessário estabelecer índices técnicos que apontem o período que a cultura possui maior exigência hídrica e a quantidade que deve ser aplicada (Tomassoni et al., 2013). A produção e a qualidade de brássicas podem ser limitadas tanto pela deficiência de água no solo como por seu excesso, o que leva à queda na produtividade dessa cultura (Tangune et al., 2016; Tomassoni et al., 2013).

Além da disponibilidade hídrica, a nutrição mineral é um fator que limita a produtividade da cultura do rabanete. $O$ nitrogênio é um macronutriente constituinte de diversos compostos orgânicos de baixo peso molecular no citoplasma, além de ser necessário para a manutenção da capacidade fotossintética, devido à sua importância na síntese de proteínas e ativação enzimática (Taiz; Zeiger, 2013; Oliveira et al., 2014).

Objetivou-se determinar 0 desempenho agronômico do rabanete em função de lâminas de irrigação e níveis de adubação nitrogenada.

\section{MATERIAL E MÉTODOS}

O experimento foi conduzido na Universidade Federal de Alagoas (UFAL) - Campus de Arapiraca, em ambiente protegido, com as coordenadas geodésicas $9^{\circ} 45^{\prime} 09^{\prime \prime}$ de latitude Sul e $36^{\circ} 39^{\prime} 40^{\prime \prime}$ de longitude Oeste e altitude de $325 \mathrm{~m}$, em período de abril a maio de 2017. Foi adotado o delineamento em blocos casualizados (DBC), em esquema fatorial $5 \times 5$ com 4 blocos, totalizando 100 parcelas experimentais. Os tratamentos foram representados por cinco lâminas de irrigação $\left(L_{1}-50 ; L_{2}-75 ; L_{3}-100 ; L_{4}\right.$ - 125 e $L_{5}-150 \%$ da Evapotranspiração da cultura (ETc)) correlacionadas à cinco níveis de adubação nitrogenada $\left(\mathrm{N}_{1}-50 ; \mathrm{N}_{2}-75 ; \mathrm{N}_{3}-100 ; \mathrm{N}_{4}-125\right.$ e $\mathrm{N}_{5}$ - 150 \% da recomendação de adubação nitrogenada para a cultura do rabanete). A unidade experimental foi representada por um recipiente plástico com área superficial de $0,0314 \mathrm{~m}^{2}$, com capacidade máxima para 5L.

A ETc foi obtida diariamente por meio do sistema SLIMCAP.App (sistema lisimétrico de informações para monitoramento do consumo de água pelas culturas) com aplicativo, Santos (2018), instalados na bancada na casa de vegetação. Para determinar o consumo, eram utilizadas provetas graduadas, diariamente, onde os valores de aplicado e drenado eram inseridos em planilhas eletrônicas e calculava a quantidade de água a ser aplicada em cada lâmina.

A adubação utilizada foi baseada pelo manual de recomendação de adubação para o estado de Pernambuco (IPA, 2008). No manual não apresenta recomendação para a cultura do rabanete, então se adotou a recomendação para a cultura do repolho, por ser da mesma família. Recomenda-se aplicar 30 e $70 \mathrm{Kg} \mathrm{ha}^{-1}$ de $\mathrm{N}$, em fundação e cobertura, respectivamente. A fonte utilizada para adubação foi a Ureia ( $45 \%$ de N). Foi distribuída a mesma quantidade dos demais nutrientes utilizados (fósforo e potássio) para ambos os tratamentos. As variáveis analisadas foram: número de folhas (NF), diâmetro do bulbo (DB), massa fresca da parte aérea (MFPA), massa seca da parte aérea (MSPA) e produtividade (PROD.). Os dados foram submetidos a análise de variância e regressão, utilizando-se 0 software estatístico SISVAR 5.6.

\section{RESULTADOS E DISCUSSÃO}

A análise de variância revelou que houve efeito significativo para o fator lâminas de irrigação para NF, MFPA, MFR, MSPA, MSR e PROD. Para os níveis de nitrogênio, apenas MFPA apresentou efeito significativo, e a interação entre ambos não foi significativa para nenhuma das variáveis analisadas (Tabela 1). Para 0 DB, Bregonci et al. (2008), trabalhando com rabanete cultivado em vaso, verificaram que o estresse hídrico reduziu o diâmetro do bulbo, diferente dos resultados encontrados no presente trabalho.

$\mathrm{O} N \mathrm{~N}$ decresceu à medida que houve aumento da lâmina de irrigação (Figura 1), semelhante aos resultados encontrados por Santos et al. (2014), na mesma região de cultivo. A redução do número de folhas ocorre de acordo com a disponibilidade hídrica, como sendo uma estratégia de sobrevivência para as plantas, que reduz o numero de folhas para que assim, ocorra diminuição da perda de água por transpiração, e consequentemente, redução da taxa fotossintética e menor crescimento (Taiz ; Zeiger, 2013). 
Tabela 1. Resumo de análise de variância e médias para número de folhas (NF), diâmetro do bulbo (DB), massa fresca da parte aérea (MFPA), massa fresca da raiz (MFR), massa seca da parte aérea (MSPA), massa seca da raiz (MSR), área foliar (AF) e produtividade (PROD.)

\begin{tabular}{|c|c|c|c|c|c|c|}
\hline \multirow{2}{*}{ Fonte de Variação } & \multirow[b]{2}{*}{ GL } & \multicolumn{5}{|c|}{ Valores dos Quadrados Médios } \\
\hline & & NF & DB & MFPA & MSPA & PROD. \\
\hline LÂMINAS (L) & 4 & $9,0 *$ & $31,3^{\mathrm{NS}}$ & $83,8 *$ & $0,9 *$ & $1,2 * *$ \\
\hline Regressão linear & 1 & $22,4 *$ & $50,1^{\mathrm{NS}}$ & $294,5 *$ & $1,8 *$ & $4381041618,0 * *$ \\
\hline Regressão quadrática & 1 & $7,9^{\mathrm{NS}}$ & $57,9^{\mathrm{NS}}$ & $24,2^{\mathrm{NS}}$ & $0,1^{\mathrm{NS}}$ & $261902285,7^{\mathrm{NS}}$ \\
\hline Regressão Cúbica & 1 & $2,2^{\mathrm{NS}}$ & $17,2^{\mathrm{NS}}$ & $8,3^{\mathrm{NS}}$ & $0,2^{\mathrm{NS}}$ & $137332164,5^{\mathrm{NS}}$ \\
\hline Desvio Regressão & 1 & $3,6^{\mathrm{NS}}$ & $0,1^{\mathrm{NS}}$ & $8,2^{\mathrm{NS}}$ & $1,4^{\mathrm{NS}}$ & $71294117,8^{\mathrm{NS}}$ \\
\hline NITROGÊNIO (N) & 4 & $1,4^{\mathrm{NS}}$ & $30,9^{\mathrm{NS}}$ & $41,6^{*}$ & $0,6^{\mathrm{NS}}$ & $22745191,5^{\mathrm{NS}}$ \\
\hline Regressão linear & 1 & $0,7^{\mathrm{NS}}$ & $10,1^{\mathrm{NS}}$ & $154,2 *$ & $1,4^{\mathrm{NS}}$ & $8644482,0^{\mathrm{NS}}$ \\
\hline Regressão quadrática & 1 & $1,7^{\mathrm{NS}}$ & $10,9^{\mathrm{NS}}$ & $6,2^{\mathrm{NS}}$ & $0,5^{\mathrm{NS}}$ & $42152320,0^{\mathrm{NS}}$ \\
\hline Regressão Cúbica & 1 & $0,6^{\mathrm{NS}}$ & $63,8^{\mathrm{NS}}$ & $4,0^{\mathrm{NS}}$ & $0,1^{\mathrm{NS}}$ & $12959140,5^{\mathrm{NS}}$ \\
\hline Desvio Regressão & 1 & $2,5^{\mathrm{NS}}$ & $38,8^{\mathrm{NS}}$ & $2,1^{\mathrm{NS}}$ & $0,3^{\mathrm{NS}}$ & $27224823,5^{\mathrm{NS}}$ \\
\hline $\mathrm{L}$ versus $\mathrm{N}$ & 16 & $2,5^{\mathrm{NS}}$ & $39,4^{\mathrm{NS}}$ & $38,8^{\mathrm{NS}}$ & $0,5^{\mathrm{NS}}$ & $10242494,0^{\mathrm{NS}}$ \\
\hline BLOCOS & 3 & $36,5^{\mathrm{NS}}$ & $916,2^{\mathrm{NS}}$ & $19,9^{\mathrm{NS}}$ & $1,8^{\mathrm{NS}}$ & $221308209,3^{\mathrm{NS}}$ \\
\hline RESÍDUO & 72 & 3,4 & 31,7 & 35,8 & 0,3 & 191323462,1 \\
\hline \multirow[t]{2}{*}{$\mathrm{CV}(\%)$} & & 22,9 & 15,4 & 28,5 & 34,2 & 25,1 \\
\hline & & unid. & $\mathrm{mm}$ & $\mathrm{g}$ & $\mathrm{g}$ & $\mathrm{Kg} \mathrm{ha}^{-1}$ \\
\hline \multicolumn{7}{|c|}{ Lâminas de Irrigação (\%) } \\
\hline 50 & & 6,9 & 34,5 & 19,1 & 1,4 & 42617,0 \\
\hline 75 & & 7,8 & 37,2 & 19,6 & 1,7 & 53813,0 \\
\hline 100 & & 8,1 & 37,8 & 20,9 & 1,6 & 55546,5 \\
\hline 125 & & 8,5 & 37,1 & 21,2 & 1,9 & 59859,0 \\
\hline 150 & & 8,7 & 37,1 & 24,3 & 1,9 & 62995,5 \\
\hline \multicolumn{7}{|l|}{ Doses de N (\%) } \\
\hline 50 & & 8,3 & 37,4 & 19,1 & 1,4 & 54212,0 \\
\hline 75 & & 8,1 & 34,5 & 19,8 & 1,7 & 56629,0 \\
\hline 100 & & 7,6 & 37,3 & 21,5 & 1,7 & 54905,5 \\
\hline 125 & & 8,1 & 37,2 & 22,1 & 1,9 & 55195,0 \\
\hline 150 & & 7,9 & 37,1 & 22,3 & 1,8 & 53889,5 \\
\hline
\end{tabular}

(*)Significativo a 5\%, ${ }^{* *}$ ) a $1 \%$ de probabilidade e (NS) Não significativo;

Figura 1. Número de folhas (unid.) em função de lâminas de irrigação (\%).

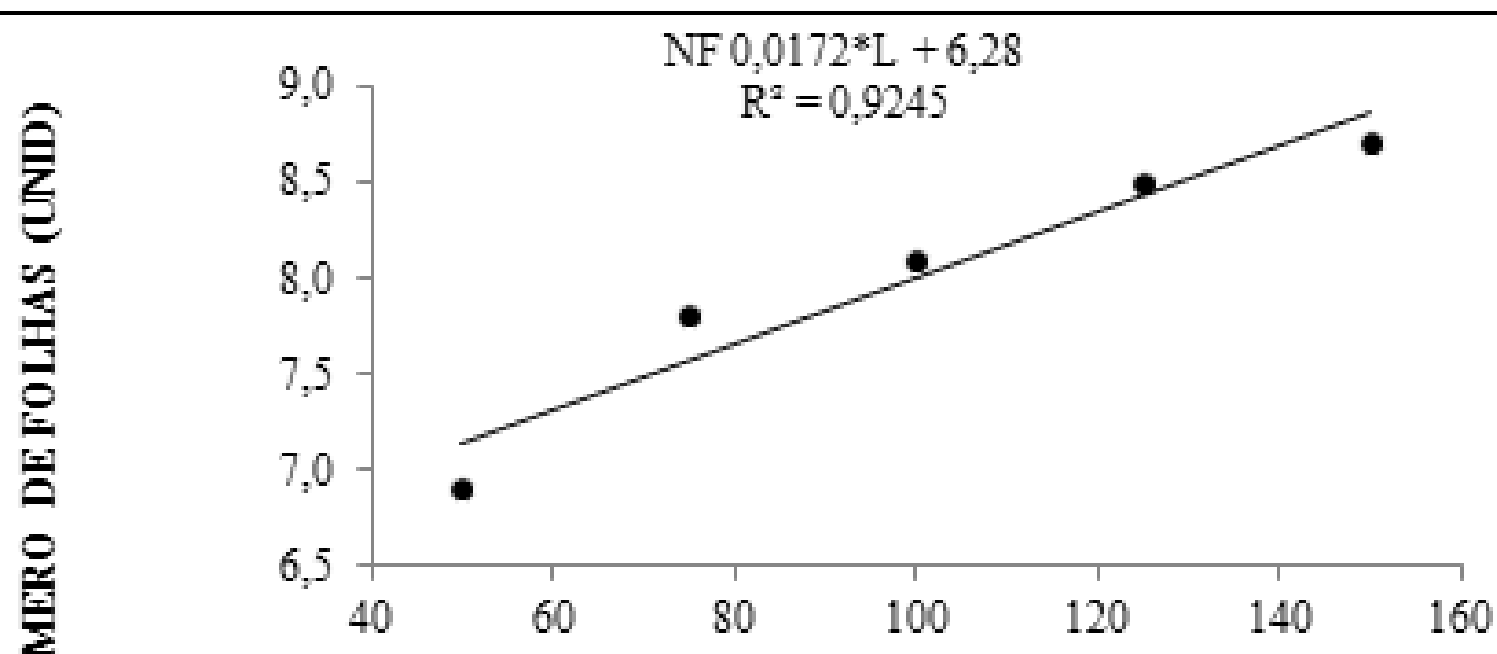

LÂMINAS DE IRRIGAC̣ÃO (\%) 
Segundo Tognon (2010), no início do estresse algumas folhas entram em processo de senescência, fato este observado pelo amarelecimento das mesmas. $O$ déficit hídrico aumenta a senescência foliar, isso ocorre porque o solo seco não pode fornecer nitrogênio suficiente para suprir as necessidades de crescimento da planta, então o nitrogênio do interior do vegetal é retranslocado das folhas mais velhas para os pontos de crescimento; entretanto, a intensidade da senescência depende da quantidade de nitrogênio no solo, das reservas de nitrogênio na planta e da demanda de nitrogênio dos pontos de crescimento (Wolfe et al., 1988).

A MFPA foi ajustada a uma função linear crescente ao amento das lâminas de irrigação e níveis de nitrogênio (Figura 2). De acordo com Paiva et al. (2005), o decréscimo de água no solo diminui o potencial de água na folha e sua condutância estomática, promovendo o fechamento dos estômatos, o que bloqueia o fluxo de $\mathrm{CO}_{2}$ para as folhas, afetando o acúmulo de fotoassimilados. Por outro lado, a planta responde positivamente às condições mais favoráveis de água no solo, mantendo taxas fotossintéticas elevadas, proporcionando uma maior produção de fotoassimilados. Para a MFPA Araújo et al. (2010) aplicando diferentes lâminas de irrigação na alface Verônica, em Boa Vista-RR encontraram um comportamento linear, semelhantes dos resultados os encontrados no presente trabalho (Figura 2A).

Figura 2. Massa fresca da parte aérea em função de lâminas de irrigação (A) e níveis de nitrogênio (B).

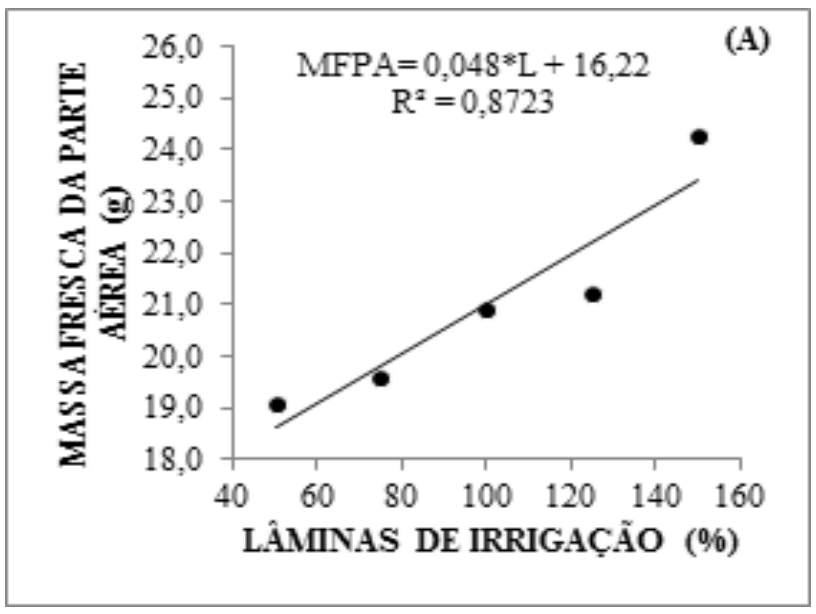

Estudos têm demonstrado que 0 acúmulo de adubação nitrogenada aumenta a resistência das culturas ao estresse hídrico (Lacerda et al., 2003; SILVA et al., 2008). Ainda, a adubação nitrogenada promove 0 crescimento das plantas (Flores et al., 2001), fazendo com

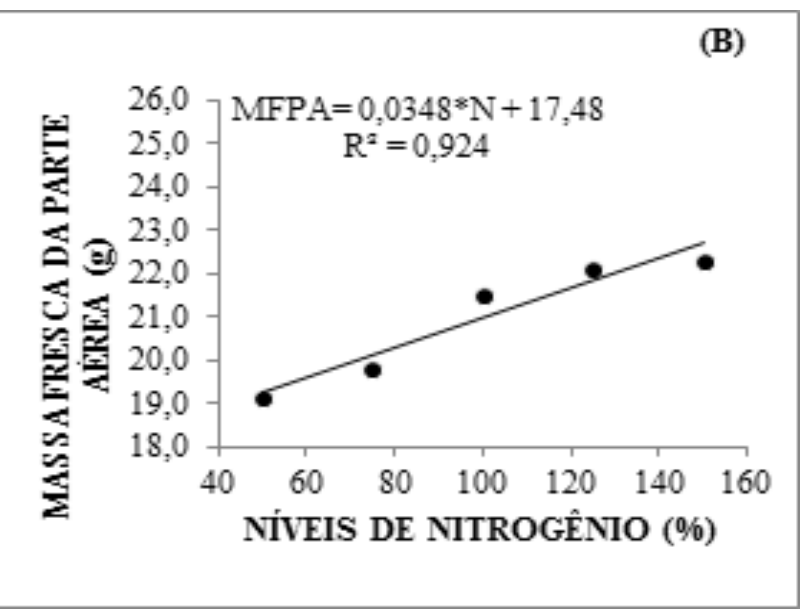

que a maior MFPA do rabanete tenha sido encontrada nos maiores níveis de adubação nitrogenada.

As maiores médias para massa seca da parte aérea e produtividade foram obtidas na maior lâmina de irrigação testada (150\% da ETc) (Figura3).

Figura 3. Massa seca da parte aérea (A) e produtividade (B) em função de lãminas de irrigação.
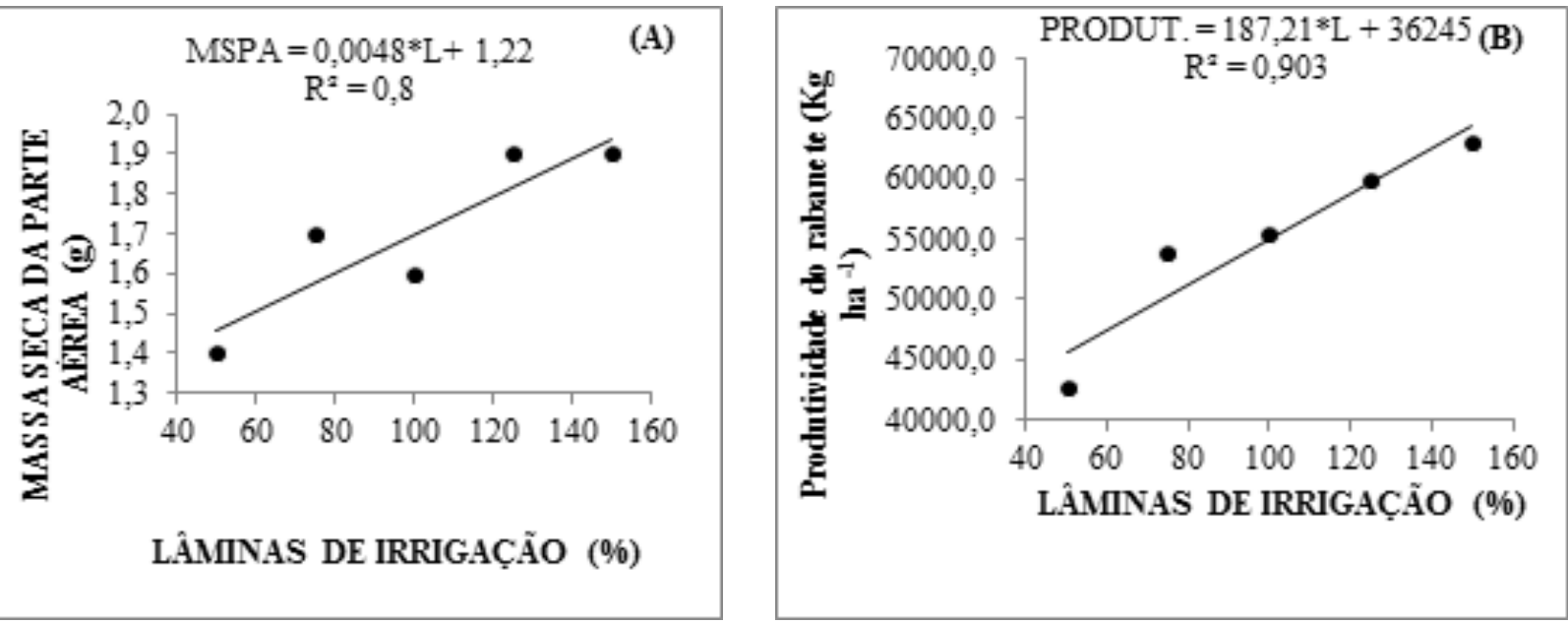
O rabanete apresenta distúrbios fisiológicos no déficit de umidade, proporcionando diferentes efeitos na quantidade e qualidade do bulbo produzido (Cunha et al., 2017). A resposta mais proeminente das plantas ao déficit hídrico, segundo Mccree ; Fernández (1989), consiste no decréscimo da produção da área foliar, do fechamento estomático, da redução caulinar, da senescência e da abscisão das folhas, culminando na redução de produção. Esses fatos podem explicar os resultados obtidos neste trabalho, quanto a pouca disponibilidade de água na fase inicial de desenvolvimento da cultura do rabanete e a sua resposta a menor lâmina de irrigação.

\section{REFERÊNCIAS BIBLIOGRÁFICA}

Araújo, W. F.; Souza, K. T. S.; Viana, T. V. A.; Azevedo, B. M.; Oliveira, G. A. Rendimento e eficiência do uso da água pela alface em função da lâmina de irrigação. Revista Caatinga, 2010, 23, 115-120.

Bregonci, I. S.; Almeida, G. D.; Brum, V. J.; Zini Júnior, A.; Reis, E. F. Desenvolvimento do sistema radicular do rabanete em condição do estresse hídrico. Idesia, 2008, 26, 1, 33-38.

Cunha, F. F.; Castro, M. A.; Godoy, A. R.; Magalhães, F. F.; Leal, A. J. F. Irrigação de cultivares de rabanete em diferentes épocas de cultivo no nordeste Sul-MatoGrossense. Irriga, 2017, 22, 3, 530-546.

Ferreira, D.F. Sisvar: a computer statistical analysis system. Ciência e Agrotecnologia, 2011, 35, 1039-1042.

Flores, P.; Carvajal, M.; Cerda, A.; Martinez, V. Salinity and ammonium/nitrate interactions on tomato plant development, nutrition, and metabolites. Journal of Plant Nutrition, 2001, 24, 10, 1561-1573.

Lacerda, C.F.; Cambraia, J.; Oliva, M.A.; Ruiz, H.A.; Prisco, J.T. Solute accumulation and distributions during shoot and development in sorghum genotypes under salt stress. Environmental and Experimental of Botany, 2003, 49, 1, 107-120.

Lima, N. B.; Alverez, H. M.; Jesus, M. A. C. L. Extração e determinação de atividade da peroxidase do rabanete em diferentes estágios de crescimento e analise da influencia dos íons cálcio, cobalto (ii) e cobre (ii) no crescimento in vitro. Anais Seminário de Iniciação Científica, 2017.

Matos, M. J. L. F.; Lana, M. M.; Santos, F. F.; Mello, M. F.; Tavares, S. A. Hortaliça: como comprar, conservar e consumir - Rabanete. 2016, Embrapa hortaliças, 2. ed. Brasilia.

Paiva, A. S.; Fernandes, E. J.; rodrigues, T. J. D.; Turco, J. E. P. Condutância estomática em folhas de feijoeiro submetido a diferentes regimes de irrigação. Revista de Engenharia Agrícola, 2005, 25, 161-169.

Pereira, M. E. M.; Lima Júnior, J. A.; Lima, V. M.; Gusmão, S. A. L.; Oliveira, P. D.; Silva, A. L. P. Viabilidade econômica da produção de couve-florirrigada por gotejamento no Nordeste Paraense. Revista Ciências Agrárias, 2018, 61, 1, 1-8.

Tangune, B. F.; Pereira, G. M.; Sousa, R. J.; Gatto, R. F. Produção de brócolis irrigado por gotejamento, sob diferentes tensões de água no solo. Semina: Ciências Agrárias, 2016, 37, 1, 7-16.

Tognon, G. B. Potencial ornamental, propagação, rendimento de óleos essenciais e resposta à deficiência hídrica de ipomeias. Passo Fundo, 2010. 133 p. Dissertação (Mestrado) - Universidade de Passo Fundo, Passo Fundo.

Santos, L. A. Sistema lisimétrico de informações para monitoramento do consumo de água pelas plantas (SLIMCAP). Arapiraca - AL, 2018. 37 p. Trabalho de Conclusão de Curso (TCC em Agronomia). Universidade Federal de Alagoas, Arapiraca.

Santos, J. C. C.; Silva, C. H.; Santos, C. S.; Silva, C. D. S.; Melo, E. B.; BARROS, A. C. Análise de crescimento e evapotranspiração da cultura do rabanete submetido a diferentes lâminas de água. Revista Verde de Agroecologia e Desenvolvimento Sustentável, 2014, 9, 1, 151-156.

Silva, E.C.; Nogueira, R.J.M.C.; Araujo, F.P.; Melo, N.F.; Azevedo Neto, A.D.; Physiological responses to salt stress in young umbu plants. Revista Brasileira de Agricultura Irrigada, 2008, 6, 2, 151-163.

Taiz, L.; Zeiger, E. Fisiologia vegetal. $4^{\circ}$ ed. Porto Alegre: Artmed, 2013.819p. 